\title{
Improved survival associated with somatic PIK3CA mutations in copy-number low endometrioid endometrial adenocarcinoma
}

\author{
DOUGLAS I. LIN
}

\author{
Department of Pathology, Beth Israel Deaconess Medical Center, Boston, MA 02215, USA
}

Received November 5, 2014; Accepted July 21, 2015

DOI: $10.3892 / 01.2015 .3702$

\begin{abstract}
The phosphoinositide-3-kinase (PI3K) signaling pathway has been implicated in the development of endometrioid endometrial adenocarcinoma (EEC). Recently, The Cancer Genome Atlas (TCGA) project stratified EEC into four molecular subgroups, with the majority of tumors falling into the copy-number low-EEC (CNL-EEC) molecular subgroup. The aim of the present study was to investigate whether alterations of the PI3K pathway are associated with specific survival outcomes in patients with EEC. The clinical and genomic data of 307 patients with endometrioid-type tumors were obtained from TCGA project, including 90 patients in the CNL-EEC subgroup. Patients were evaluated in terms of survival and clinicopathological characteristics, as well as mutations in the PI3K catalytic subunit alpha (PIK3CA) gene and their effect on PIK3CA function. In CNL-EEC subgroup patients, somatic PIK3CA mutations (48/90 cases) were associated with significantly improved overall survival compared with that of wild-type PIK3CA $(\mathrm{P}=0.018)$. Furthermore, this improved survival was specific to the CNL-EEC subgroup and was not observed in other TCGA molecular subgroups. The majority of CNL-EEC cases were low-stage (stage I) and low-to-intermediate grade (grades 1-2) endometrioid tumors. There were no significant differences in age, stage, histology or International Federation of Gynecology and Obstetrics grade between $P I K 3 C A$-mutated and non-mutated patient groups $(\mathrm{P}>0.05)$. In addition, the majority of cases contained activating PIK3CA mutations. Overall, in the TCGA cohort, PIK3CA mutations had a favorable effect on the survival of patients with EEC, and this effect was dependent on tumoral molecular sub-stratification. Future studies on larger independent cohorts with long term follow-up are warranted to further analyze this association.
\end{abstract}

Correspondence to: Dr Douglas I. Lin, Department of Pathology, Beth Israel Deaconess Medical Center, East Building 121, 330 Brookline Avenue, Boston, MA 02215, USA

E-mail: dilin@bidmc.harvard.edu

Key words: endometrial adenocarcinoma, PIK3CA mutation, phosphoinositide-3-kinase pathway, The Cancer Genome Atlas

\section{Introduction}

Endometrial adenocarcinoma is the most frequently diagnosed type of gynecological malignancy, with 280,000 cases reported worldwide in 2008 (1). In the United States, it is estimated that there were $\sim 40,000$ new cases per year, with 7,500 mortalities reported in 2008 (2). The majority of cases are grade 1 endometrioid-type adenocarcinoma confined to the uterus for which total hysterectomy and bilateral salpingo-oophorectomy is standard treatment $(1,2)$. However, a small but significant percentage of cases recur following total hysterectomy. This recurrence, or cases that are metastatic upon presentation, exhibit a limited response to systemic therapy (chemotherapy, hormonal therapy or radiation). The five-year survival rate is $>95 \%$ for women with disease confined to the uterus (stage I) and $\sim 15 \%$ for distant, high-stage disease (3). Recently, The Cancer Genome Atlas (TCGA) project stratified endometrioid endometrial adenocarcinoma (EEC) into four different molecular subgroups, each with distinct survival outcomes: i) Copy-number low (CNL) alterations; ii) copy-number high alterations (serous-like); iii) polymerase $\varepsilon$ (POLE) ultramutated; and iv) microsatellite instability hypermutated (4). In this stratification system, the CNL-EEC molecular subgroup exhibited intermediate progression-free survival compared with the POLE ultramutated (best prognosis) and copy-number high, serous-like (worst prognosis) tumors (4).

Similar to previous studies, which revealed a high percentage of somatic phosphatase and tensin homolog (PTEN), phosphoinositide-3-kinase catalytic subunit alpha (PIK3CA) and $\mathrm{PI} 3 \mathrm{~K}$ regulatory subunit $1(P I K 3 R I)$ mutations in EECs, the TCGA study revealed that a significant number of endometrial adenocarcinomas had genetic alterations in the PI3K/AKT pathway, suggesting a potential for targeted therapy with specific inhibitors (4-11). More specifically, TCGA demonstrated that the PI3K pathway was altered in 92\% (83/90) CNL-EEC cases, as evidenced by somatic mutations in the PTEN, PIK3CA and PIK3R1 genes (4). The majority of CNL-EEC tumors (77\%; 69/90) exhibited somatic PTEN mutations, while approximately half $(53 \%$; 48/90) exhibited somatic PIK3CA mutations and one-third $(33 \% ; 30 / 90)$ contained somatic PIK3R1 mutations. Furthermore, approximately two-thirds of tumors $(68 \%$; 61/90) exhibited somatic mutations in $>1$ member of the PI3K pathway (4). These and previous findings (4-11) support the hypothesis that activation of the PI3K signaling pathway is an important step in the development of EEC. 
The aim of the present study was to investigate whether mutations of PI3K pathway members are associated with specific survival outcomes in patients with endometrial adenocarcinomas and whether these outcomes are specific to a particular molecular subgroup.

\section{Materials and methods}

Survival analysis. The clinical data of 307 newly diagnosed endometrial carcinoma patients with endometrioid-type histology, including 90 cases of CNL-EEC, was obtained from TCGA Research Network (4) and integrated with PIK3CA mutation data from TCGA Data Portal Open-Access directory (https://tcga-data.nci.nih.gov/tcga/). Overall and disease-free survival analysis were then performed using the cBio Cancer Genomics Portal (http://cbioportal.org). The present study was approved by the Beth Israel Deaconess Medical Center-HCC Institutional Review Board.

Review of pathological data. Pathology reports were downloaded from TCGA Data Portal Open-Access directory or via the cBio Cancer Genomics Portal. Quality control hematoxylin and eosin (H\&E) images from frozen and permanent sections were re-analyzed by a board-certified gynecological pathologist (Dr Douglas Lin) at the Department of Pathology, Beth Israel Deaconess Medical Center (Boston, MA, USA) to determine that tumors were indeed of the endometrioid histological subtype. Re-analysis was performed via TCGA BioSig website (http://tcga.lbl.gov:8080/biosig/tcgadownload.do) hosted by Lawrence Berkeley National and via the Cancer Digital Slide Archive (http://cancer.digitalslidearchive.net; Emory University, Atlanta, GA, USA). Tumor stage (12) and overall International Federation of Gynecology and Obstetrics (FIGO) grade (13) were also independently re-evaluated where possible, however, this was deferred to the available pathology reports, as only limited virtual images from each case were publicly available for review.

Analysis of PIK3CA mutations. Somatic PIK3CA mutations derived from Next Generation exome sequencing were retrieved from TCGA Data Portal Open-Access directory via the cBio Portal (http://www.cbioportal.org/public-portal/). The effect of specific somatic mutations on PIK3CA function was determined by cross-referencing them with previously published studies, wherein the effect of specific PIK3CA mutations on p110- $\alpha$ activation or gain-of-function was demonstrated in vitro or in vivo $(9,14-16)$. p110- $\alpha$ is the protein encoded by the PIK3CA gene and represents the catalytic subunit of PI3K. The presence of specific PIK3CA somatic mutations in other cancer types was determined by analyzing the Catalogue of Somatic Mutations in Cancer database (http://cancer.sanger.ac.uk/cancergenome/projects/cosmic/). All websites were accessed between July 1, 2013 and May 1, 2014.

Statistical analysis. Kaplan-Meier overall and disease-free survival curves and corresponding P-values were calculated using the cBio Cancer Genomics Portal (http://cbioportal.org; Memorial Sloan Kettering Cancer Center, New York, NY, USA) with significance estimated by log-rank test $(17,18)$.
Table I. Clinicopathological characteristics of patients in The Cancer Genome Atlas copy-number low endometrioid endometrial carcinoma molecular subgroup with and without somatic PIK3CA mutations.

\begin{tabular}{lccc}
\hline & \multicolumn{2}{c}{ PIK3CA } & \\
\cline { 2 - 3 } Characteristic & Non-mutated & Mutated & P-value \\
\hline Cases, $\mathrm{n}$ & 42 & 48 & \\
Age, years & & & 0.902 \\
Range & $37-90$ & $34-89$ & \\
Mean & 60.6 & 60.3 & \\
Median & 59 & 60 & \\
Stage, n $(\%)$ & & & 0.406 \\
I & $34(81.0)$ & $40(83.3)$ & \\
II & $1(2.3)$ & $2(4.0)$ & \\
III & $6(14.3)$ & $6(13.0)$ & \\
IV & $1(2.3)$ & $0(0.0)$ & \\
Grade, n $(\%)$ & & & \\
1 & $22(52.4)$ & $23(47.9)$ & \\
2 & $18(42.9)$ & $21(43.8)$ & \\
3 & $2(4.8)$ & $4(8.3)$ & \\
\hline
\end{tabular}

Statistical analysis of clinicopathological characteristics (age and tumor stage and grade) were assessed using the t-test and paired t-test, respectively. $\mathrm{P}<0.05$ was considered to indicate a statistically significantly difference.

\section{Results}

Clinical data from 307 newly diagnosed endometrial carcinoma patients with endometrioid-type histology, including 90 cases of the CNL-EEC subtype, was obtained from TCGA Research Network (4) and integrated with PIK3CA mutation data from TCGA Data Portal via the cBio Cancer Genomics Portal $(4,17,18)$. Survival analysis of all TCGA tumors with endometrioid-type histology $(n=307)$, regardless of molecular subtype, revealed that patients with somatic PIK3CA mutations exhibited significantly improved overall survival and marginally prolonged progression-free survival compared with those possessing wild-type PIK3CA (log-rank test $\mathrm{P}=0.001$ and $\mathrm{P}=0.077$, respectively; Fig. $1 \mathrm{~A}$ and $\mathrm{B}$ ).

The overall survival outcome difference was specific to the CNL-EEC patients ( $\mathrm{n}=90 ; \mathrm{P}=0.018$; Fig. $1 \mathrm{C}$ and $\mathrm{D})$, as no significant association with overall and disease-free survival was observed in the three other TCGA molecular subgroups (copy-number high, $\mathrm{P}=0.442$ and $\mathrm{P}=0.225$; $P O L E$ ultramutated, no mortality or recurrence; or microsatellite instability hypermutated tumors, $\mathrm{P}=0.108$ and $\mathrm{P}=0.949$ ) (data not shown). For this reason, the remainder of the study was focused on the CNL-EEC subgroup. In CNL-EEC, the mean follow-up times for patients with mutated and wild-type $P I K 3 C A$ were 33 and 26 months, respectively. The median follow-up times for patients with mutated PIK3CA and wild-type PIK3CA were 29 and 21 months, respectively. There were no reported mortalities among patients with PIK3CA mutations (0\%; 

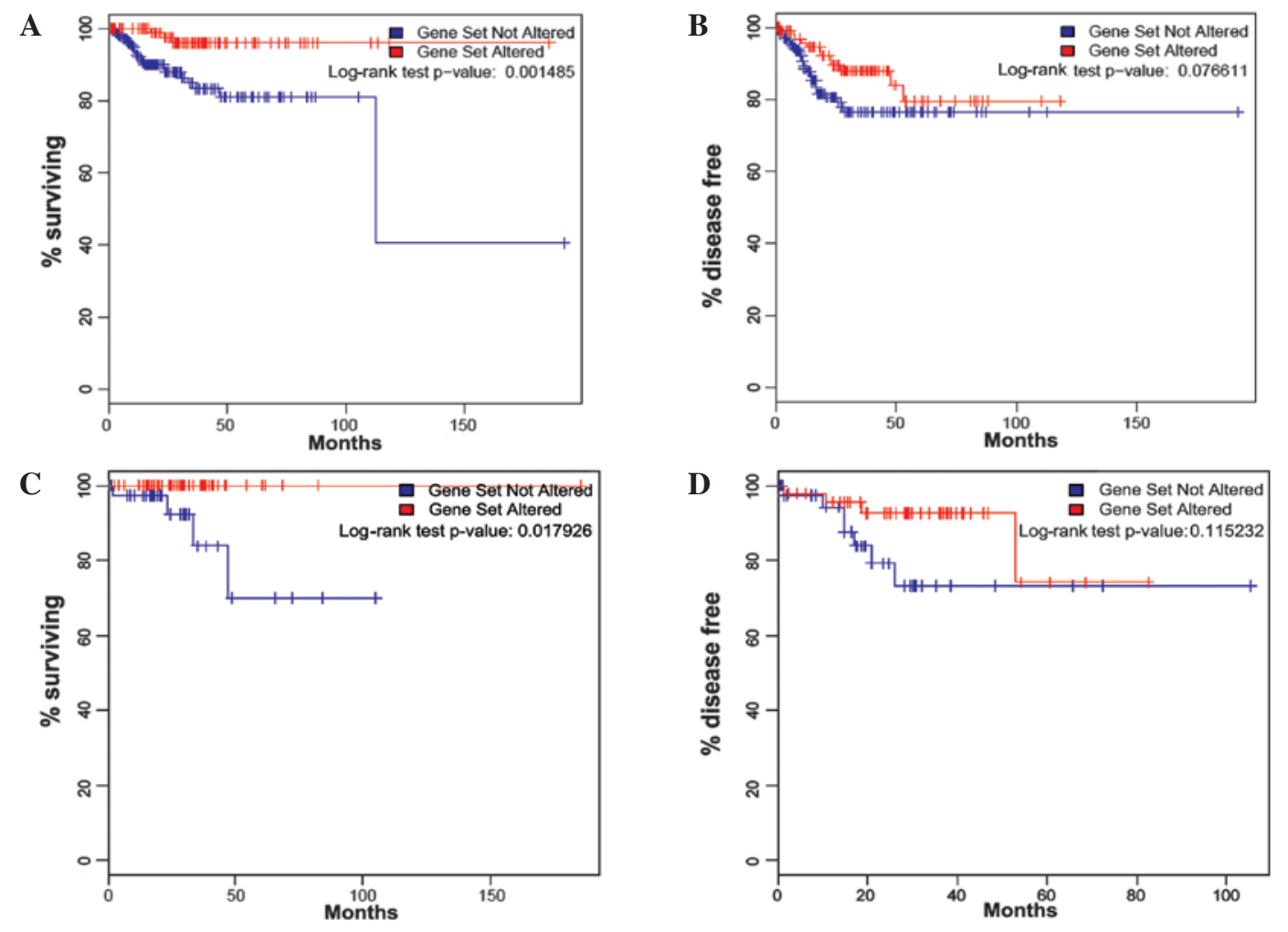

Figure 1. Kaplan-Meier survival curves for PIK3CA-mutated tumors. (A) Overall and (B) disease-free survival of all TCGA patients with endometrioid-type tumors (n=307), regardless of molecular sub-stratification, with (red) or without (blue) PIK3CA mutations. (C) Overall and (D) disease-free survival of TCGA patients in the copy-number low endometrioid endometrial adenocarcinoma molecular subgroup (n=90) with (red) or without (blue) PIK3CA mutations. TCGA, The Cancer Genome Atlas.

A

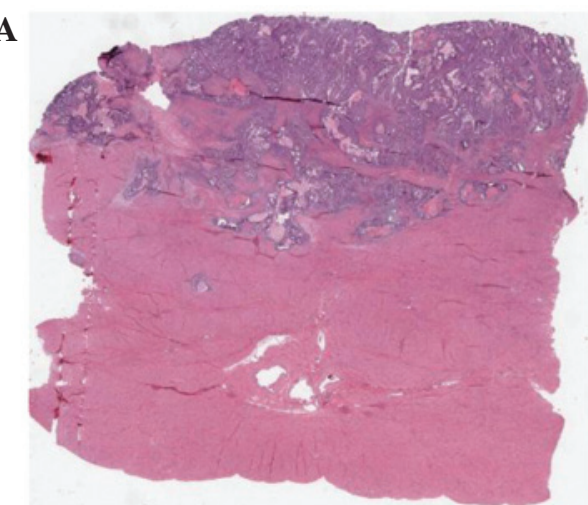

C

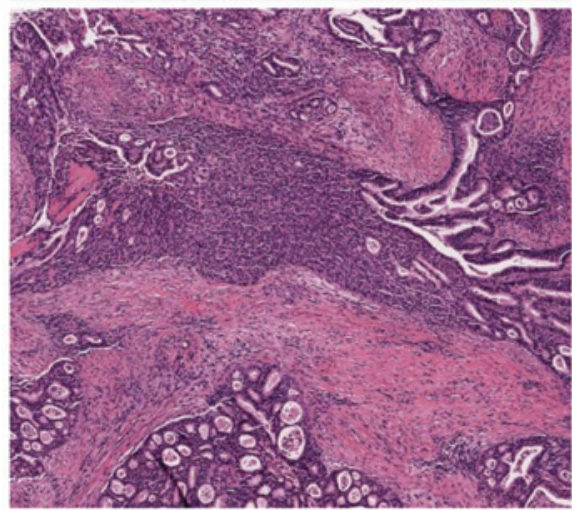

B

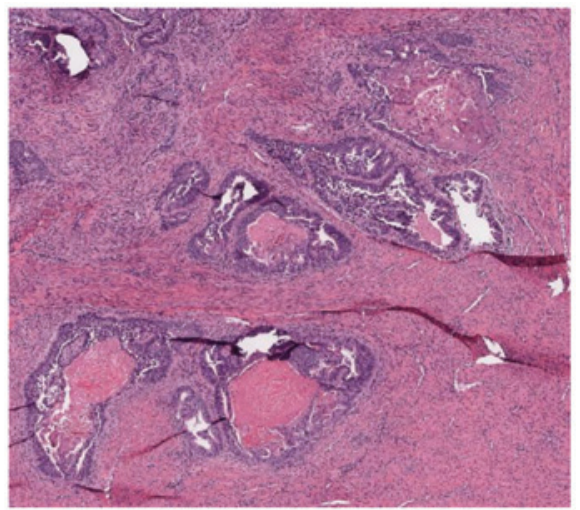

D

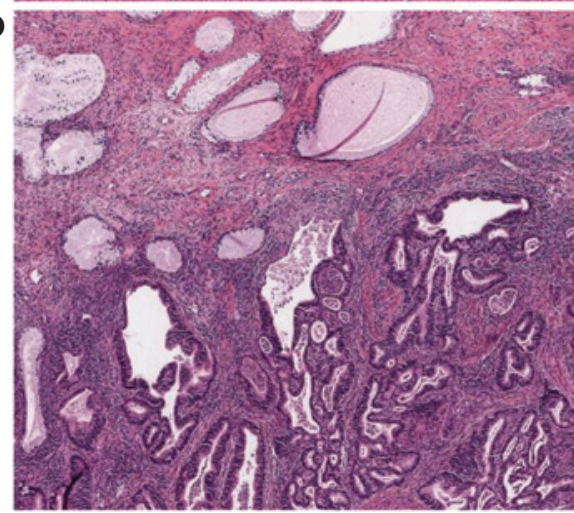

Figure 2. Histology of PIK3CA-mutated tumors (hematoxylin and eosin staining) from patients in TCGA. Patient no. TCGA-D1-A16D: (A) Full-thickness permanent section of well-differentiated tumor, demonstrating stage I myometrial invasion (magnification, x10). (B) Higher magnification of the same tumor characterized by well-formed, endometrioid-type glands and no significant nuclear atypia [FIGO grade 1 (of 3)] (magnification, x40). Patient no. TCGA-D1-A16J: (C) Endometrioid endometrial adenocarcinoma, characterized by cribriform and solid growth patterns [FIGO grade 2 (of 3)] (magnification, x40). (D) Different section of the same tumor involving stage II endocervical stroma (magnification, x40). Images and data obtained from the cBio Cancer Genomics Portal (http:// cbioportal.org). TCGA, The Cancer Genome Atlas; FIGO, International Federation of Gynecology and Obstetrics. 
Table II. Summary of somatic PIK3CA mutations in the copy-number low endometrioid endometrial adenocarcinoma subgroup.

\begin{tabular}{|c|c|c|c|c|c|}
\hline Mutation & $\mathrm{n}$ & $\begin{array}{l}\text { Effect on } \\
\text { function }\end{array}$ & Type & Domain & $\begin{array}{c}\text { Present in } \\
\text { other cancers }\end{array}$ \\
\hline WGIHLMPP11del & 1 & Unknown & IF del & $\mathrm{ABD}$ & No \\
\hline R88Q & 2 & Activating & Missense & $\mathrm{ABD}$ & Yes \\
\hline R93Q & 2 & Activating & Missense & $\mathrm{ABD}$ & Yes \\
\hline I102del & 1 & Unknown & IF del & $\mathrm{ABD}$ & No \\
\hline N107S & 1 & Unknown & Missense & $\mathrm{ABD}$ & No \\
\hline $\mathrm{K} 111 \mathrm{E}$ & 1 & Activating & Missense & $\mathrm{ABD}$ & Yes \\
\hline L113del & 1 & Unknown & IF del & $\mathrm{ABD}$ & Yes \\
\hline R115L & 1 & $\mathrm{a}$ & Missense & $\mathrm{ABD}$ & Yes \\
\hline G118D & 1 & Activating & Missense & $\mathrm{ABD}$ & Yes \\
\hline V344M & 1 & Unknown & Missense & $\mathrm{C} 2$ & Yes \\
\hline N345K & 2 & Activating & Missense & $\mathrm{C} 2$ & Yes \\
\hline C420R & 2 & Activating & Missense & $\mathrm{C} 2$ & Yes \\
\hline 463_465GSN $>$ D & 1 & Unknown & IF del & $\mathrm{C} 2$ & No \\
\hline E542K & 5 & Activating & Missense & Helical & Yes \\
\hline E542A & 1 & Activating & Missense & Helical & Yes \\
\hline E545K & 8 & Activating & Missense & Helical & Yes \\
\hline E545A & 1 & Activating & Missense & Helical & Yes \\
\hline E545G & 1 & Activating & Missense & Helical & Yes \\
\hline E545D & 1 & $\mathrm{a}$ & Missense & Helical & Yes \\
\hline Q546P & 2 & Activating & Missense & Helical & Yes \\
\hline Q546K & 1 & Activating & Missense & Helical & Yes \\
\hline Q546R & 1 & $\mathrm{a}$ & Missense & Helical & Yes \\
\hline C901F & 1 & Unknown & Missense & Kinase & Yes \\
\hline D939G & 2 & Unknown & Missense & Kinase & Yes \\
\hline G1007R & 1 & Unknown & Missense & Kinase & Yes \\
\hline M1043V & 2 & Activating & Missense & Kinase & Yes \\
\hline M1043I & 2 & Activating & Missense & Kinase & Yes \\
\hline H1047R & 7 & Activating & Missense & Kinase & Yes \\
\hline H1047L & 2 & Activating & Missense & Kinase & Yes \\
\hline H1047Q & 1 & $\mathrm{a}$ & Missense & Kinase & Yes \\
\hline
\end{tabular}

Six tumors exhibited $>1$ somatic mutation. a, mutation occurring in a position previously implicated in PIK3CA activation; ABD, adaptor-binding domain; C2, protein kinase-C homology 2; IF del, in-frame deletion.

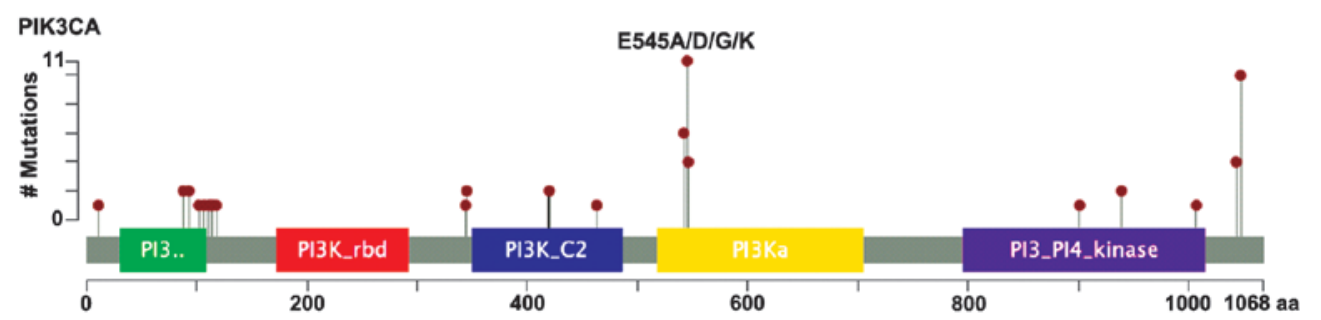

Figure 3. Distribution of somatic PIK3CA mutations in the copy-number low endometrioid endometrial adenocarcinoma molecular subgroup. Mutations occurred predominantly in hotspots centered on the following PIK3CA functional domains: Adaptor-binding domain (green), protein kinase-C homology 2 (blue), helical (yellow) and kinase (purple). PI3K, phosphoinositide-3-kinase.

0/48), while 4 mortalities were reported among the patients with wild-type PIK3CA $(9.5 \% ; 4 / 42)$. Of the patients with somatic PIK3CA mutations, 5 exhibited recurrent disease $(10.4 \%$; 5/48), while 8 patients with wild-type $P I K 3 C A$ had recurrent disease $(19.0 \% ; 8 / 42)$.
Next, the pathological data was re-reviewed in terms of patient age, tumor type, stage and FIGO grade via the tools available at TCGA Data Portal Open-Access directory and the cBio portal. Patient age in PIK3CA-mutated cases ranged from 34-89 years (mean, 60.3 years; median, 60 years), which was 
similar to that of the non-mutated cases (range, 37-90 years; mean, 60.6 years; median, 59 years) (Table I). The majority of PIK3CA-mutated cases were of stage I (83\%; 40/48) and FIGO grade 1 or 2 disease (48 and 44\%, respectively) (Table I and Fig. 2). There were no significant differences in age, tumor stage and FIGO grade between PIK3CA-mutated and non-mutated cancers (Table I). For all cases in the CNL-EEC subgroup, the tumors were of endometrioid histological type. This was confirmed by the presence of back to back atypical endometrial type glands with complex architecture, no intervening stroma and varying differentiation upon review of available $\mathrm{H} \& \mathrm{E}$ images (Fig. 2).

Pathological analysis of 3 PIK3CA-mutated cases also demonstrated the presence of a serous carcinoma component. However, the images that were available for review were more consistent with endometrioid-type tumors. In addition, these 3 cases exhibited no TP53 mutations or homozygous deletion, indicating that the histological type was not serous carcinoma. These findings suggest that survival differences between PIK3CA mutated and non-mutated cases may not be attributed to differences in patient age, tumor stage, FIGO grade or histological subtype.

A total of 56 somatic PIK3CA mutations were identified in 48 patients with CNL-EEC, and a subset of these mutated tumors $(12.5 \%$; 6/48) contained $>1$ PIK3CA mutation. PIK3CA mutations occurred predominantly in hotspots centered on p110- $\alpha$ functional domains (Fig. 3), and around 30\% (17/56) occurred within exons $1-7$, which encode the N-terminal domains of p110- $\alpha$, including the p85/adaptor-binding domain and the protein kinase-C homology 2 domain. In addition, around $70 \%(39 / 56)$ of PIK3CA mutations occurred within exons 9 and 20, which encode the C-terminal helical and kinase domains (Fig. 3). The majority of cases (79\% of patients and $75 \%$ of overall mutations) contained mutations implicated in PIK3CA activation and gain-of-function (Table II). The following recurrent activating mutations were also identified: R88Q, R93Q, N345K, C420R, E542K, E545K (most common), Q546P, D939G, M1043V, M1043I, H1047R (second most common) and H1047L (Fig. 3 and Table II). These findings support the hypothesis that endometrial adenocarcinomas possess a unique spectrum of somatic PIK3CA mutations in which a significant number of mutations occur in the amino terminal domain of p110- $\alpha$ compared with other cancer types, such as colorectal, breast or bladder carcinomas $(4,9)$.

\section{Discussion}

Despite its growth-promoting properties, the effect of PIK3CA mutation on patient prognosis varies according to the cancer subtype. In patients with colorectal or lung cancer, mutations of PIK3CA are associated with poor prognosis. By contrast, PIK3CA mutations have been associated with favorable prognosis in patients with breast cancer and esophageal squamous cell carcinomas (19-22). In endometrial cancer patients, the influence of PIK3CA mutations on survival and prognosis has been unclear, as there is data to suggest an association between PIK3CA mutations and both favorable and unfavorable prognoses (23-25).

The TCGA Data Portal on endometrial adenocarcinoma offers a host of tools that can be used to interrogate specific genetic events relative to survival and clinicopathological characteristics, as well as to generate hypotheses for future testing. In the present study, we hypothesized that $P I K 3 C A$ mutations in EEC may be associated with favorable survival outcomes, possibly specific to a molecularly sub-defined cohort of tumors that exhibit low somatic copy-number alterations and microsatellite stability. The significantly improved overall survival observed in all patients with endometrioid-type adenocarcinomas exhibiting PIK3CA mutations, regardless of the molecular subtype, may reflect the fact that the majority of TCGA tumors were in the CNL-EEC subgroup.

However, due to the limitations of the TCGA study (i.e., small sample size of the CNL-EEC subgroup, relatively short follow-up time, lack of information on co-morbidities), these findings are exploratory and hypothesis-generating in nature, rather than definitive. For this reason, future studies with multivariate analyses and long-term follow-up to determine the effect of other clinical co-variables (including race, body mass index, estrogen receptor/progesterone receptor status, co-morbidities and treatment regimens) on survival outcome are required. In addition, long-term multivariate analyses with increased power are essential to determine the effect of other molecular co-variables. For example, Liang et al (11) previously reported that ARIDIA mutations co-occur with PI3K pathway mutations in endometrial cancer and lead to activation of the PI3K signaling pathway. In the TCGA CNL-EEC subgroup, somatic mutations of ARIDIA occurred in $42 \%$ of cases $(38 / 90)$ with a trend towards improved overall survival $(\mathrm{P}=0.0517)$ and no differences in progression-free survival $(\mathrm{P}=0.86)$. By contrast, significantly improved overall survival was observed in cases with PIK3CA-ARIDIA co-occurring mutations $(\mathrm{P}=0.00026)$, with no differences in progression-free survival $(\mathrm{P}=0.88$; data not shown).

Finally, the mechanism by which PIK3CA mutations affect patient survival and the mechanism that explains why there appears to be no association between $P I K 3 R 1$ mutations and survival outcome are unclear, as PIK3CA and PIK3RI are subunits of the same kinase $(8,11)$. One possible explanation for this is that $P I K 3 C A$ - and $P I K 3 R I$-mutated tumors may have different driver mutations.

In conclusion, the results presented in the current study suggest that PIK3CA mutations may serve as a favorable prognostic biomarker in EEC, depending on further molecular sub-stratification of the tumors. Therefore, future studies on larger independent cohorts with long term follow-up are warranted to further analyze this association and to validate the findings of the TCGA project.

\section{Acknowledgements}

The present study was performed in accordance with the The Cancer Genome Atlas (TCGA) publication policy for endometrial carcinomas, as outlined in TCGA data portal (http://cancergenome.nih.gov). In addition, the current study has been previously published as an abstract for the 2015 United States and Canadian Academy of Pathology annual meeting (www.nature.com/modpathol/journal/v27/n2s/pdf/ modpathol201415a.pdf). 


\section{References}

1. Ferlay J, Shin HR, Bray F, Forman D, Mathers C and Parkin DM: Estimates of worldwide burden of cancer in 2008: GLOBOCAN 2008. Int J Cancer 127: 2893-2917, 2010

2. Jemal A, Siegel R, Ward E, Hao Y, Xu J, Murray T and Thun MJ: Cancer statistics, 2008. CA Cancer J Clin 58: 71-96, 2008.

3. Siegel R1, DeSantis C, Virgo K, Stein K, Mariotto A, Smith T, Cooper D, Gansler T, Lerro C, Fedewa S, et al: Cancer treatment and survivorship statistics, 2012. CA Cancer J Clin 62: 220-241, 2012.

4. Kandoth C, Schultz N, Cherniack AD, Akbani R, Liu Y, Shen H, Robertson AG, Pashtan I, Shen R, Benz CC, et al; Cancer Genome Atlas Research Network: Integrated genomic characterization of endometrial carcinoma. Nature 497: 67-73, 2013.

5. Risinger JI, Hayes AK, Berchuck A and Barrett JC: PTEN/MMAC1 mutations in endometrial cancers. Cancer Res 57: 4736-4738, 1997.

6. Oda K, Stokoe D, Taketani Y and McCormick F: High frequency of coexistent mutations of PIK3CA and PTEN genes in endometrial carcinoma. Cancer Res 65: 10669-10673, 2005.

7. Shoji K, Oda K, Nakagawa S, Hosokawa S, Nagae G, Uehara Y, Sone K, Miyamoto Y, Hiraike H, Hiraike-Wada O, et al: The oncogenic mutation in the pleckstrin homology domain of AKT1 in endometrial carcinomas. Br J Cancer 101: 145-148, 2009.

8. Urick ME, Rudd ML, Godwin AK, Sgroi D, Merino M and Bell DW: PIK3R1 (p85 $\alpha$ ) is somatically mutated at high frequency in primary endometrial cancer. Cancer Res 71: 4061-4067, 2011.

9. Rudd ML, Price JC, Fogoros S, Godwin AK, Sgroi DC, Merino MJ and Bell DW: A unique spectrum of somatic PIK3CA (p110 $\alpha)$ mutations within primary endometrial carcinomas. Clin Cancer Res 17: 1331-1340, 2011.

10. Cheung LW, Hennessy BT, Li J, Yu S, Myers AP, Djordjevic B, Lu Y, Stemke-Hale K, Dyer MD, Zhang F, et al: High frequency of PIK3R1 and PIK3R2 mutations in endometrial cancer elucidates a novel mechanism for regulation of PTEN protein stability. Cancer Discov 1: 170-185, 2011.

11. Liang H, Cheung LW, Li J, Ju Z, Yu S, Stemke-Hale K, Dogruluk T, Lu Y, Liu X, Gu C, et al: Whole-exome sequencing combined with functional genomics reveals novel candidate driver cancer genes in endometrial cancer. Genome Res 22: 2120-2129, 2012

12. Edge S, Byrd D, Compton C, et al (eds): AJCC Cancer Staging. Manual. 7th edition. Springer, New York, NY, 2010.

13. Silverberg SG, Kurman RJ, Nogales F, et al: Tumors of the uterine corpus: Epithelial tumours and related conditions. In: Tumors of the Breast and Gynecologic Tract. Tavassoli FA and Devilee PL (eds). IARC Press, Lyon, pp218, 2002.

14. Burke JE, Perisic O, Masson GR, Vadas O and Williams RL: Oncogenic mutations mimic and enhance dynamic events in the natural activation of phosphoinositide 3-kinase p110 $\alpha$ (PIK3CA). Proc Natl Acad Sci USA 109: 15259-15264, 2012.
15. Gymnopoulos M, Elsliger MA and Vogt PK: Rare cancer-specific mutations in PIK3CA show gain of function. Proc Natl Acad Sci USA 104: 5569-5574, 2007.

16. Rios JJ, Paria N, Burns DK, Israel BA, Cornelia R, Wise CA and Ezaki M: Somatic gain-of-function mutations in PIK3CA in patients with macrodactyly. Hum Mol Genet 22: 444-451, 2013.

17. Cerami E, Gao J, Dogrusoz U, Gross BE, Sumer SO, Aksoy BA, Jacobsen A, Byrne CJ, Heuer ML, Larsson E, et al: The cBio cancer genomics portal: An open platform for exploring multidimensional cancer genomics data. Cancer Discov 2: 401-404, 2012.

18. Gao J, Aksoy BA, Dogrusoz U, Dresdner G, Gross B, Sumer SO, Sun Y, Jacobsen A, Sinha R, Larsson E, et al: Integrative analysis of complex cancer genomics and clinical profiles using the cBioPortal. Sci Signal 6: pl1, 2013.

19. Liao X, Morikawa T, Lochhead P, Imamura Y, Kuchiba A, Yamauchi M, Nosho K, Qian ZR, Nishihara R, Meyerhardt JA, et al: Prognostic role of PIK3CA mutation in colorectal cancer: Cohort study and literature review. Clin Cancer Res 18: 2257-2268, 2012.

20. Zhang L, Shi L, Zhao X, Wang Y and Yue W: PIK3CA gene mutation associated with poor prognosis of lung adenocarcinoma. Onco Targets Ther 6: 497-502, 2013.

21. Kalinsky K, Jacks LM, Heguy A, Patil S, Drobnjak M, Bhanot UK, Hedvat CV, Traina TA, Solit D, Gerald W, et al: PIK3CA mutation associates with improved outcome in breast cancer. Clin Cancer Res 15: 5049-5059, 2009.

22. Shigaki H, Baba Y, Watanabe M, Murata A, Ishimoto T, Iwatsuki M, Iwagami S, Nosho K and Baba H: PIK3CA mutation is associated with a favorable prognosis among patients with curatively resected esophageal squamous cell carcinoma. Clin Cancer Res 19: 2451-2459, 2013.

23. McIntyre JB, Nelson GS, Ghatage P, Morris D, Duggan MA, Lee CH, Doll CM and Köbel M: PIK3CA missense mutation is associated with unfavorable outcome in grade 3 endometrioid carcinoma but not in serous endometrial carcinoma. Gynecol Oncol 132: 188-193, 2014

24. Catasus L, Gallardo A, Cuatrecasas M and Prat J: PIK3CA mutations in the kinase domain (exon 20) of uterine endometrial adenocarcinomas are associated with adverse prognostic parameters. Mod Pathol 21: 131-139, 2008.

25. Dong Y, Yang X, Wong O, Zhang X, Liang Y, Zhang Y, Wong W, Nong L, Liao Q and Li T: PIK3CA mutations in endometrial carcinomas in Chinese women: Phosphatidylinositol 3'-kinase pathway alterations might be associated with favorable prognosis. Hum Pathol 43: 1197-1205, 2012. 